\title{
REDUCTIONS AND CONTRACTIONS OF 1-LOOP TENSOR FEYNMAN INTEGRALS*
}

\author{
Ievgen Dubovyk, Janusz Gluza \\ Institute of Physics, University of Silesia \\ Uniwersytecka 4, 40-007 Katowice, Poland \\ Andrea A. Almasy, Tord Riemann \\ Deutsches Elektronen-Synchrotron, DESY \\ Platanenallee 6, 15738 Zeuthen, Germany
}

(Received November 11, 2013)

\begin{abstract}
Dedicated to the memory of our late Colleague and Friend Jochem Fleischer (1937-2013). The present work is based on a decade of common fruitful research, which would have been impossible without his impetus, imagination and dedication
\end{abstract}

We report on the progress in constructing contracted one-loop tensors. Analytic results for rank $R=4$ tensors, cross-checked numerically, are presented for the first time.

DOI:10.5506/APhysPolB. 44.2241

PACS numbers: $11.80 . \mathrm{Cr}, 12.38 . \mathrm{Bx}$

\section{Introduction}

There are a few source-open programs for 5- and 6-point reductions: LoopTools/FF $(n \leq 5$, rank $\leq 4)-$ T. Hahn [1, 2], Golem95 - T. Binoth et al. [3], PJFry $(n \leq 5$, rank $\leq 5)-$ V. Yundin et al. [4, 5]. Some of these packages need, in addition, a library of scalar functions: 't Hooft, Veltman [6], LoopTools/FF, QCDloop/FF - K. Ellis and G. Zanderighi $[2,7]$ or OneLOop with complex masses — van Hameren [8]. In most cases, these packages suffice to calculate one-loop processes, however, there are at least two reasons for improvements. First, which is always desirable, speed

* Presented by I. Dubovyk and T. Riemann at the XXXVII International Conference of Theoretical Physics "Matter to the Deepest" Ustroń, Poland, September 1-6, 2013. 
improvements. This is important when calculations are included into precise measurements using Monte Carlo methods. As already discussed in [9], available methods are at the edge of applications at low energy calculations. Second, not all of them are able to fulfil high demands concerning accuracy at the very specific kinematic points; an example has been shown in [10].

We are working on independent calculations for tensor contractions as an alternative to the PJFry reductions [11], based on work by DavydychevTarasov-Fleischer-Jegerlehner-Riemann-Yundin (DTFJRY) [12-16]. First numerical studies have been discussed in [17]. In this material, we report on analytic results for rank 4 and comment on improvements concerning the OLEC package [18].

\section{Contractions for the 5-point functions with rank $R=4$}

We present here for the first time, results of contracted tensors of rank $R=4$

$$
I_{5}^{\mu \nu \lambda \rho}=I_{5}^{\mu \nu \lambda} \cdot Q_{0}^{\rho}-\sum_{s=1}^{5} I_{4}^{\mu \nu \lambda, s} \cdot Q_{s}^{\rho},
$$

for lower rank results, see $[15,16]$. After contraction with chords $q$ (differences of external momenta), we get

$$
q_{a, \mu} q_{b, \nu} q_{c, \lambda} q_{d, \rho} I_{5}^{\mu \nu \lambda \rho}=C E_{4, a b c d}=-\frac{1}{2} C E_{3, a b c}, Y_{d}+C_{5, a b c d} .
$$

Here,

$$
Q_{s}^{\rho}=\sum_{i=1}^{5} q_{i}^{\rho} \frac{\left(\begin{array}{l}
s \\
i
\end{array}\right)_{5}}{()_{5}}, \quad s=0, \ldots, 5
$$

The first term $q_{a, \mu} q_{b, \nu} q_{c, \lambda} I_{5}^{\mu \nu \lambda}$ is known [17], the second term has to be determined

$$
C_{5, a b c d}=-\sum_{s=1}^{5} q_{a \mu} q_{b \nu} q_{c \lambda} I_{4}^{\mu \nu \lambda, s} \frac{1}{2}\left(\delta_{d s}-\delta_{5 s}\right),
$$

and it becomes

$$
\begin{aligned}
& C_{5, a b c d}=\frac{1}{16}\left\{G^{5}+\delta_{a b} \delta_{a c} \delta_{a d} G^{d}-I_{1}^{5 a b c}-I_{1}^{5 a b d}-I_{1}^{5 a c d}-I_{1}^{5 b c d}+I_{1}^{a b c d}\right. \\
& -J_{3}^{a 5}-J_{3}^{b 5}-J_{3}^{c 5}-J_{3}^{d 5}+R^{5 a b}+R^{5 a c}+R^{5 b c}+R^{5 d a}+R^{5 d b}+R^{5 d c} \\
& +\delta_{b c} \delta_{b d}\left(J_{3}^{a d}-J_{3}^{5 d}\right)+\delta_{a c} \delta_{a d}\left(J_{3}^{b d}-J_{3}^{5 d}\right)+\delta_{a b} \delta_{a d}\left(J_{3}^{c d}-J_{3}^{5 d}\right)
\end{aligned}
$$




$$
\begin{aligned}
& +\delta_{a b} \delta_{a c}\left(J_{3}^{d c}-J_{3}^{5 c}\right)+\delta_{a b} \delta_{c d} \tilde{J}_{3}^{d b}+\delta_{a d} \delta_{b c} \tilde{J}_{3}^{d c}+\delta_{a c} \delta_{b d} \tilde{J}_{3}^{d c} \\
& +\delta_{a b}\left(\tilde{J}_{3}^{5 b}-R^{b 5 c}-R^{b d 5}+R^{b d c}\right)+\delta_{a c}\left(\tilde{J}_{3}^{5 c}-R^{c 5 b}-R^{c d 5}+R^{c d b}\right) \\
& +\delta_{a d}\left(\tilde{J}_{3}^{d 5}-R^{d 5 b}-R^{d 5 c}+R^{d b c}\right)+\delta_{b c}\left(\tilde{J}_{3}^{5 c}-R^{c 5 a}-R^{c d 5}+R^{c d a}\right) \\
& +\delta_{b d}\left(\tilde{J}_{3}^{d 5}-R^{d 5 a}-R^{d 5 c}+R^{d a c}\right)+\delta_{c d}\left(\tilde{J}_{3}^{d 5}-R^{d 5 a}-R^{d 5 b}+R^{d a b}\right) \\
& +\delta_{a b} \delta_{a d} J_{4}^{d} Y_{c}+\delta_{a c} \delta_{a d} J_{4}^{d} Y_{b}+\delta_{b c} \delta_{b d} J_{4}^{d} Y_{a}+\delta_{a b}\left(R^{b d}-R^{b 5}\right) Y_{c} \\
& +\delta_{a c}\left(R^{c d}-R^{c 5}\right) Y_{b}+\delta_{a d}\left(R^{d c}-R^{d 5}\right) Y_{b}+\delta_{a d}\left(R^{d b}-R^{d 5}\right) Y_{c} \\
& +\delta_{b c}\left(R^{c d}-R^{c 5}\right) Y_{a}+\delta_{b d}\left(R^{d c}-R^{d 5}\right) Y_{a}+\delta_{b d}\left(R^{d a}-R^{d 5}\right) Y_{c} \\
& +\delta_{c d}\left(R^{d a}-R^{d 5}\right) Y_{b}+\delta_{c d}\left(R^{d b}-R^{d 5}\right) Y_{a}+\left(I_{4}^{d}-I_{4}^{5}\right) Y_{a} Y_{b} Y_{c} \\
& +\left(I_{3}^{c d}-I_{3}^{5 c}-I_{3}^{5 d}+R^{5}+\delta_{c d} R^{d}\right) Y_{a} Y_{b} \\
& +\left(I_{3}^{b d}-I_{3}^{5 b}-I_{3}^{5 d}+R^{5}+\delta_{b d} R^{d}\right) Y_{a} Y_{c} \\
& +\left(I_{3}^{a d}-I_{3}^{5 a}-I_{3}^{5 d}+R^{5}+\delta_{a d} R^{d}\right) Y_{b} Y_{c} \\
& +\left(I_{2}^{b c d}-I_{2}^{5 b c}-I_{2}^{5 b d}-I_{2}^{5 c d}-J_{4}^{5}+R^{5 b}+R^{5 c}+R^{5 d}\right) Y_{a} \\
& +\left(I_{2}^{a c d}-I_{2}^{5 a c}-I_{2}^{5 a d}-I_{2}^{5 c d}-J_{4}^{5}+R^{5 a}+R^{5 c}+R^{5 d}\right) Y_{b} \\
& \left.+\left(I_{2}^{a b d}-I_{2}^{5 a b}-I_{2}^{5 a d}-I_{2}^{5 b d}-J_{4}^{5}+R^{5 a}+R^{5 b}+R^{5 d}\right) Y_{c}\right\},
\end{aligned}
$$

where we have introduced

$$
\begin{aligned}
J_{3}^{s t} & \equiv \frac{1}{\left(\begin{array}{c}
s t \\
s t
\end{array}\right)_{5}}\left\{-\left(\begin{array}{l}
s \\
s
\end{array}\right)_{5} I_{3}^{[d+], s t}+\left(\begin{array}{l}
t s \\
0 s
\end{array}\right)_{5} R^{t s}-\sum_{u=1}^{5}\left(\begin{array}{l}
t s \\
u s
\end{array}\right)_{5} R^{t s u}\right\}, \\
\tilde{J}_{3}^{s t} & \equiv \frac{1}{\left(\begin{array}{l}
s t \\
s t
\end{array}\right)_{5}}\left\{\left(\begin{array}{l}
s \\
t
\end{array}\right)_{5} I_{3}^{[d+], s t}+\left(\begin{array}{c}
s t \\
0 t
\end{array}\right)_{5} R^{t s}-\sum_{u=1}^{5}\left(\begin{array}{l}
s t \\
u t
\end{array}\right)_{5} R^{t s u}\right\}, \\
G^{s} & \equiv \frac{1}{\left(\begin{array}{l}
s \\
s
\end{array}\right)_{5}}\left\{-2()_{5} R^{[d+], s}+\left(\begin{array}{l}
s \\
0
\end{array}\right)_{5} J_{4}^{s}-\sum_{t=1}^{5}\left(\begin{array}{l}
s \\
t
\end{array}\right)_{5} J_{3}^{t s}\right\} .
\end{aligned}
$$

$J_{4}^{s}$ and $R^{[d+], s}$ are given in Eqs. (2.24) and (2.44) of [15], respectively. For further abbreviations, see (2.24), (2.49), (2.9), (2.17), (2.34), (2.41) of [15]. There also $R^{s}, R^{s t}, R^{t s u}$ are defined, $Y_{a}=Y_{a 5}-Y_{55}, Y_{a b}=-\left(q_{a}-q_{b}\right)^{2}+$ $m_{a}^{2}+m_{b}^{2}$. 
Some numerical results for a 5-point function with rank $R=4$ are added in [18]. For scalar functions, we use the OneLOop package and compare the results with LoopTools/FF. For the considered kinematic points, full agreement has been obtained. The kinematics is that of the process $e^{-} e^{+} \rightarrow$ $\mu^{-} \mu^{+} \gamma$. Using an MC generator, we have checked thousands of points up to rank three. All of them agreed between OLEC and LT/OneLOop. For ranks 3 and the new results presented here, we made checks also against the public version of Golem95, available at http://golem.hepforge.org/95/. At the OLEC webpage [18], we added two sets of files with the output. In set I, the kinematics is chosen such that 3-point functions hit the IR singularities, while in set II, the 3-point functions are slightly off the IR singularities. The Golem95 results were different from LT, OneLOop and OLEC, starting already from rank $3^{1}$.

For rank four, we give below such an example:

$\begin{array}{ll}\mathrm{p} 1 \mathrm{~s}=1.1163688400000000 \mathrm{E}-002 & \mathrm{p} 2 \mathrm{~s}=2.6109999999999998 \mathrm{E}-007 \\ \mathrm{p} 3 \mathrm{~s}=0.0000000000000000 & \mathrm{p} 4 \mathrm{~s}=2.6109999999999998 \mathrm{E}-007 \\ \mathrm{p} 5 \mathrm{~s}=1.1163688400000000 \mathrm{E}-002 & \\ \mathrm{~s} 12=-0.70858278190000001 & \mathrm{~s} 23=-1.5343299000000002 \mathrm{E}-003 \\ \mathrm{~s} 34=-0.12851860429999998 & \mathrm{~s} 45=-0.61023937949999996 \\ \mathrm{~s} 15=0.92668942420000000 & \mathrm{~m} 1 \mathrm{~s}=1.1163688361676107 \mathrm{E}-002 \\ \mathrm{~m} 2 \mathrm{~s}=0.0000000000000000 & \mathrm{~m} 3 \mathrm{~s}=2.6112003932088364 \mathrm{E}-007 \\ \mathrm{~m} 4 \mathrm{~s}=2.6112003932088364 \mathrm{E}-007 & \mathrm{~m} 5 \mathrm{~s}=0.0000000000000000\end{array}$

The $\mathrm{R}=4$ contractions, $a, b, c, d=3,3,3,3$

OLEC: $(-48094.107454542318 \quad,-47802.087465035322)$

LoopTools: ( $-48094.107465 \quad,-47802.0874605 \quad)$

The $R=4$ contractions, $a, b, c, d=3,3,3,4$

OLEC: ( -18463.1204 $24842149 \quad,-23446.470412257226)$

LoopTools: ( $-18463.120431 \quad,-23446.470409)$

The $\mathrm{R}=4$ contractions, $\mathrm{a}, \mathrm{b}, \mathrm{c}, \mathrm{d}=3,3,3,5$

OLEC: ( $0.0000000000000000 \quad, 0.0000000000000000)$

LoopTools $(0.0000000000000000,0.0000000000000000)$

The last result with $d=5$ is a virtue of the construction of chords, where $q_{5}=0$.

\section{OLEC package, Fortran code}

The idea of external contractions has been implemented for the first time in the $\mathrm{C}++$ code OLEC for tensors up to rank 3 [17], and basic examples

\footnotetext{
${ }^{1}$ The problem in Golem95 v.1.2.1 has been settled in the meantime for the set I with
} changelog 128 (11 Oct, 2013), https://golem.hepforge.org/trac/changeset/128 
are given at [18]. In the meantime, a Fortran code has been written with the aim of further optimization. The calculation of contracted tensor integrals consists of two basic steps. The first and most time consuming step is a preparation of building blocks for a calculation - basic scalar integrals $(\sim 50 \%)$ and the so-called signed minors $(\sim 25 \%)$. We cannot make much about scalar integral libraries, unless new independent developments appear. For some recent efforts in this direction, see [19]. However, the calculation of signed minors can be improved, both the cache system and the computation algorithm. The second step is performing of the contractions $(\sim 25 \%)$, which also can be improved. During the initialization procedure, scalar integrals and signed minors are calculated and stored in the RAM memory. For an efficient usage of memory, appropriate data structures are constructed. E.g. for 5-point kinematics, we have, in total, only 30 different basic scalar integrals, while for signed minors situation it is more complicated because all minors with up to 4 scratched rows and columns are needed. The "natural" storage model for such objects in the form of multidimensional arrays is not an optimal solution. For example, for minors of rank 3 (3 rows and columns are excluded), one has an array with 46656 elements but according to the minors' symmetry properties, only 210 of them are different and nonzero. For the purpose of minimal memory usage, a linear storage model is chosen. In general, minors of a given rank are stored in the memory as one-dimensional arrays and access to them can be done according to the following pattern: $\operatorname{minor}_{\text {rank }}[\operatorname{AddressTable}[\operatorname{func}(i, j, k \ldots)]]$, as it was first done in [11]. For a given minor indices $i, j, k, \ldots$, a function 'func' based on bitwise operations calculates an address of an element from the constant table 'AddressTable'. This table is the same for all minors and contains positions of values of minors in array 'minor ${ }_{\text {rank }}$ '. Finally, the cache contains a few hundred of double precision numbers for all building blocks like minors, scalar integrals and auxiliary functions.

In the new version of the library, the computation algorithm for signed minors has also been changed. In practical computations of one-loop crosssections, usually at least all contractions up to rank 3 are needed (like in QED). It means that minors of all ranks are needed as well. The procedure is iterative, cache is filled first from minors of the highest rank 4 (with 8 indices) which are used further to calculate minors of rank 3. And so on. All loops connected with minor indices during this procedure are unrolled and the highest level of optimization is applied for compilation (these are algebraical manipulations for which "-O3" optimization does not spoil double accuracy). Unrolling and usage of optimalization for algebraic part of the package allows to decrease extremely the matrix algebra computation time. 


\section{Summary and outlook}

In recent years, the strategy described has been developed in a bunch of papers for explicit analytical and recursive treatment of heptagon, hexagon and pentagon tensor integrals of rank $R$ in terms of pentagons and boxes of rank $R-1$. A systematic derivation of expressions which are explicitly free of inverse Gram determinants ( $)_{5}$ until pentagons of rank $R=5$ has been worked out. The numerical package OLEC for contracted tensor integrals in $\mathrm{C}++$ and Fortran $[18,20]$ are under development and tests.

Our preliminary benchmarks show that the OLEC library in the present form is faster than 5-point tensor reductions implemented in LoopTools/FF by about an order of magnitude (tested up to rank 3). Work in progress includes a correct treatment of small Gram determinant cases for the reductions (expansion in small parameters [11] or using hypergeometric representations [21]), adding rank 4 (this paper) and rank 5 contractions for 5 -point functions, programming contracted tensors for 6- and 7-point functions.

Work supported by the European Initial Training Network LHCPHENOnet PITN-GA-2010-264564.

\section{REFERENCES}

[1] T. Hahn, M. Perez-Victoria, Comput. Phys. Commun. 118, 153 (1999) [arXiv:hep-ph/9807565].

[2] G. van Oldenborgh, Comput. Phys. Commun. 66, 1 (1991); http://www.xs4all.nl/ gjvo/

[3] T. Binoth et al., Comput. Phys. Commun. 180, 2317 (2009) [arXiv:0810.0992 [hep-ph]].

[4] V. Yundin, "Massive loop corrections for collider physics", Ph.D. Thesis, Humboldt-Universität zu Berlin, 2012, see http://edoc.hu-berlin.de/docviews/abstract.php?id=39163 and http://urn:nbn:de:kobv:11-100199626

[5] J. Fleischer, T. Riemann, V. Yundin, in: [22], preprint DESY 11-252, 2011, http://www-library.desy.de/cgi-bin/showprep.pl?desy11-252

[6] G. 't Hooft, M. Veltman, Nucl. Phys. B153, 365 (1979).

[7] R.K. Ellis, G. Zanderighi, J. High Energy Phys. 02, 002 (2008) [arXiv:0712.1851 [hep-ph]].

[8] A. van Hameren, Comput. Phys. Commun. 182, 2427 (2011) [arXiv: 1007.4716 [hep-ph]].

[9] M. Gunia, see transparencies of talk at this conference, http://indico.if.us.edu.pl/event/us2013 
[10] J. Gluza, M. Gunia, T. Riemann, M. Worek, PoS RADCOR2011, 034 (2011) [arXiv: 1201.0968 [hep-ph]].

[11] V. Yundin, C++ package PJFry, available at https://github.com/Vayu/PJFry/

[12] A.I. Davydychev, Phys. Lett. B263, 107 (1991).

[13] O. Tarasov, Phys. Rev. D54, 6479 (1996) [arXiv:hep-th/9606018].

[14] J. Fleischer, F. Jegerlehner, O. Tarasov, Nucl. Phys. B566, 423 (2000) [arXiv:hep-ph/9907327].

[15] J. Fleischer, T. Riemann, Phys. Rev. D83, 073004 (2011) [arXiv:1009.4436 [hep-ph]].

[16] J. Fleischer, T. Riemann, Phys. Lett. B701, 646 (2011) [arXiv:1104.4067 [hep-ph]].

[17] J. Fleischer et al., PoS LL2012, 051 (2012) [arXiv:1211.3921 [hep-ph]].

[18] A.A. Almasy et al., OLEC project; $\mathrm{C}++$ and Mathematica packages OLEC v.0.9, available at the Silesian Univ., Katowice, webpage http://prac.us.edu.pl/ gluza/olec/

[19] J.P. Guillet, E. Pilon, M. Rodgers, M. Zidi, arXiv:1310.4397 [hep-ph].

[20] A.A. Almasy, J. Fleischer, J. Gluza, T. Riemann, publication in preparation; A.A. Almasy, "Fortran package for rank 5 tensor integrals", available on request.

[21] J. Fleischer, F. Jegerlehner, O. Tarasov, Nucl. Phys. B672, 303 (2003) [arXiv:hep-ph/0307113].

[22] J. Alcaraz Maestre et al., Report of the SM and NLO Multileg and SM MC Working Groups for the Workshop "Physics at TeV Colliders", Les Houches, France, 31 May-8 June, 2011, see arXiv:1203.1488 [hep-ph] and arXiv:1203.6803 [hep-ph]. 